\title{
Mesh Encircling Total Extraperitoneal (TEP) Repair of Inguinal Hernia without a Fixation Material or Preformed Mesh: Overlay Closure of Slit Without Additional Mesh
}

\author{
Dong Jin Park, M.D., Yeong Cheol Im, M.D., Sung Soo Yang, M.D., Gyu Yeol Kim, M.D. \\ Department of Surgery, Ulsan University Hospital, University of Ulsan College of Medicine, Ulsan, Korea
}

\begin{abstract}
Purpose: Mesh non-fixation in TEP is associated with lower cost and shorter operative time, and it is safe and recommended when performed by an experienced surgeon. In performance of TEP surgery using a non-fixation mesh, particularly by a non-experienced surgeon, prevention of mesh migration is an important issue for mesh application. The aim of this study was to report on a fixation method using a non-preformed mesh encircling a cord structure without the use of tacks, staples, or fibrin sealants in TEP surgery.
\end{abstract}

Methods: A total of 41 patients who had undergone mesh-encircling TEP without fixation from December 2008 (first case of surgery) to June 2012 were analyzed.

Results: The mean follow-up period was 23.2 months (12 35 months). Three patients complained of scrotal discomfort, and one patient complained of scrotal edema, but they were resolved with conservative management. There was no recurrence during the follow up period.

Conclusion: The method of mesh-encircling TEP without fixation material and no additional mesh in which the slit of the mesh is wrapped around the cord structure using an overlay suture is a simple and safe technique without compromising recurrence or chronic pain.

Keywords: TEP, Inguinal hernia, Fixation, Mesh

This is an Open Access article distributed under the terms of the Creative Commons Attribution Non-Commercial License (http:// creativecommons.org/licenses/by-nc/3.0/) which permits unrestricted non-commercial use, distribution, and reproduction in any medium, provided the original work is properly cited.

\author{
Received September 5, 2014 \\ Revised 1st October 13, 2014 \\ 2nd October 15, 2014 \\ Accepted October 15, 2014 \\ Corresponding author \\ Yeong Cheol Im \\ Department of Surgery, Ulsan \\ University Hospital, University of \\ Ulsan College of Medicine, Jeonha- \\ dong, Dong-gu, Ulsan 682-714, \\ Korea \\ Tel: +82-52-250-7109 \\ Fax: +82-52-250-7108 \\ E-mail: imyc0323@gmail.com
}

Copyright @ 2015 The Journal of Minimally Invasive Surgery. All rights reserved.

\section{INTRODUCTION}

Since the 1990s, laparoscopic total extraperitoneal repair (TEP) of inguinal hernia has been performed widely.1 The advantages of laparoscopic inguinal hernia repair include an earlier return to work and a shorter hospital stay than with open surgery. ${ }^{1-3}$ When TEP is performed, the mesh fixation methods vary, including tack, staple or fibrin sealant fixation, as well as non-fixation methods. ${ }^{4.5}$ However, these tools are expensive and do not have insurance coverage. Mesh non-fixation in TEP is associated with lower cost and shorter operative time, ${ }^{4}$ and it is safe and recommended when done by an experienced surgeon. ${ }^{5}$ Some reports have revealed complications of mesh migration after hernia repair, and inadequate mesh fixation is a contributing factor to hernia recurrence due to mesh migration and the erosion of surrounding tissue. ${ }^{6-8}$ When TEP surgery is done using a non-fixation mesh, especially by a non-experienced surgeon, the prevention of mesh migration is an important issue for mesh application. We report a fixation method using a nonpreformed mesh encircling around a cord structure without the use of tacks, staples or fibrin sealants in TEP surgery. 


\section{MATERIALS AND METHODS}

We retrospectively reviewed the medical records of 75 patients of laparoscopic mesh herniorrhaphy for inguinal hernia from December 2008 ( $1^{\text {st }}$ case of surgery) to June 2012, and we searched for patients that experienced meshencircling TEP without fixation. At the beginning, we performed a transabdominal preperitoneal hernia repair using a covering mesh with tacks in sixteen patients, and these patients were excluded. Four patients that had no record of the mesh application method were excluded, as were fourteen patients that had undergone TEP with a covering mesh, not an encircling mesh. A total of 41 patients that had undergone mesh-encircling TEP without fixation were analyzed. After discharge, the patients were followed up 2 to 4 weeks later for detection of recurrence or complications, and we instructed the patients to immediately revisit our hospital whenever lower abdominal or scrotal pain was felt or recurrence was suspected. In January 2013, we contacted the 41 patients by phone and gained information about whether or not recurrence or inguinal pain occurred. And, we reviewed records about operation time, hospital stay, postoperative visual analogue scale, postoperative analgesic use of hospital day, and complications.

\section{Creation of extraperitoneal space}

Standardized TEP was always carried out under general anesthesia. A 2 to $3-\mathrm{cm}$ infraumbilical skin incision was made, and the ipsilateral anterior rectus sheath was identified and opened. The rectus muscle was retracted laterally. Blunt dissection was carried out using the index finger to create an extraperitoneal space above the arcuate line of the posterior rectus sheath. With the index finger inserted into

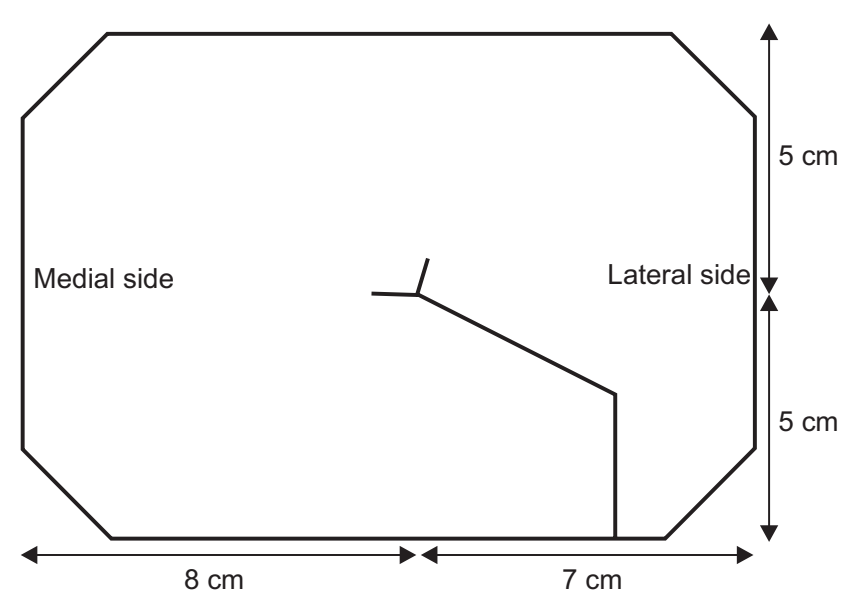

Fig. 1. Mesh design. the extraperitoneal space, a 5-mm bladeless trocar was used to puncture at the point of the index finger tip at the midpoint between the symphysis pubis and the umbilicus. A $12-\mathrm{mm}$ cannula for a camera was inserted through the infraumbilical incision. A maximum of $15 \mathrm{mmHg}$ of carbon dioxide insufflation was done, followed by sharp dissection using an endo-dissector inserted through the 5-mm cannula, and an extraperitoneal space was made in the Retzius space.

Another $12-\mathrm{mm}$ cannula was inserted at 1 to $2 \mathrm{~cm}$ above the symphysis pubis, and sharp dissection into the areolar tissue plane was done between the inferior epigastric vessel and peritoneum toward the ipsilateral lower quadrant area. The arcuate line of the posterior rectus sheath was detached from the anterior rectus sheath without tearing the peritoneum. After dissection of the extraperitoneal space, exposing the obturator foramen, the symphysis pubis, and the anterior superior iliac spine, the hernia sac was identified and separated from the spermatic cord structure until the testis vessel, vas deferens, and peritoneal surface formed an isosceles triangle. The spermatic cord structure was fully separated from the external iliac vessel after complete detachment of the hernia sac.

\section{Mesh application}

A $15 \times 10 \mathrm{~cm}$ multifilament polypropylene mesh (Surgipro $^{\mathrm{TM}}$, Covidien $^{\mathrm{TM}}$, MA, USA) was used, and the detailed mesh design is shown in Fig. 1. The designed mesh was inserted into the extraperitoneal space, and the slit of the mesh encircled the cord structure. The slit of the mesh was closed by an overlapping suture using non-absorbable thread (black silk $3-0)$ near the cord structure to make a new internal inguinal

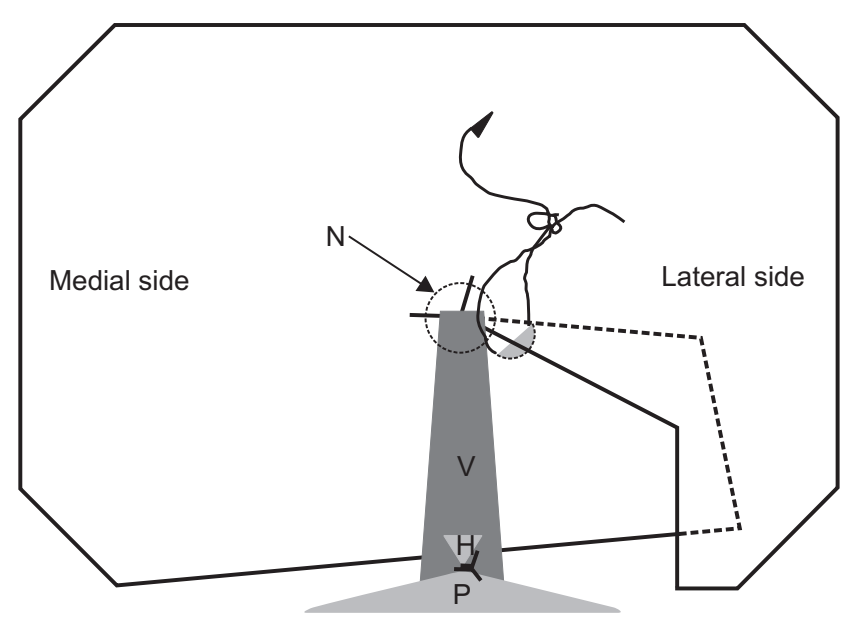

Fig. 2. Applied mesh for male. $N=$ new internal inguinal ring; $V=$ vas deferens and testicular vessels; $\mathrm{H}=$ ligated hernia sac; $\mathrm{P}=$ peritoneum; $S=$ suture ligation of mesh encircling. 


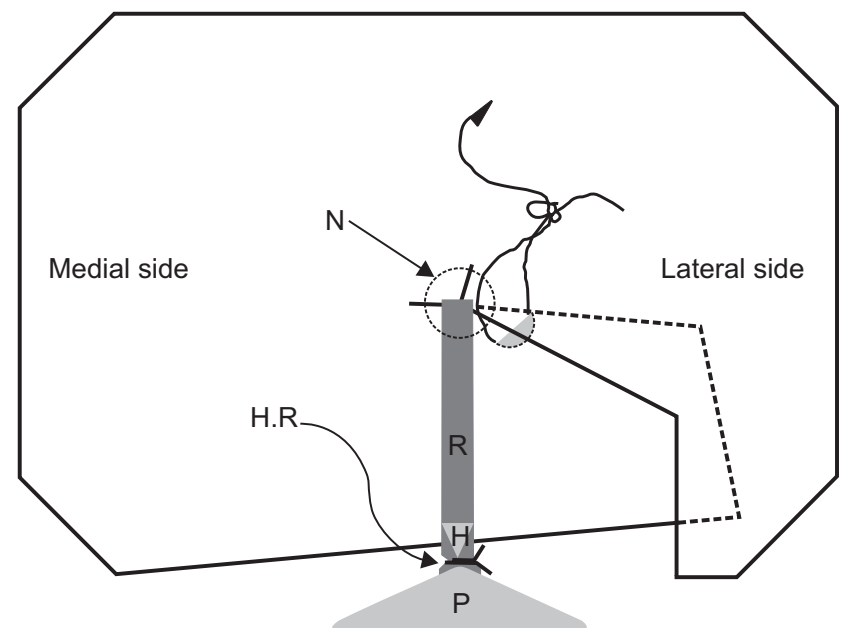

Fig. 3. Applied mesh for female. $N=$ new internal inguinal ring; $R=$ round ligament; $H$ = ligated hernia sac; $P$ = peritoneum; $S=$ suture ligation of mesh encircling; H.R = hernia sac was ligated with round ligament.

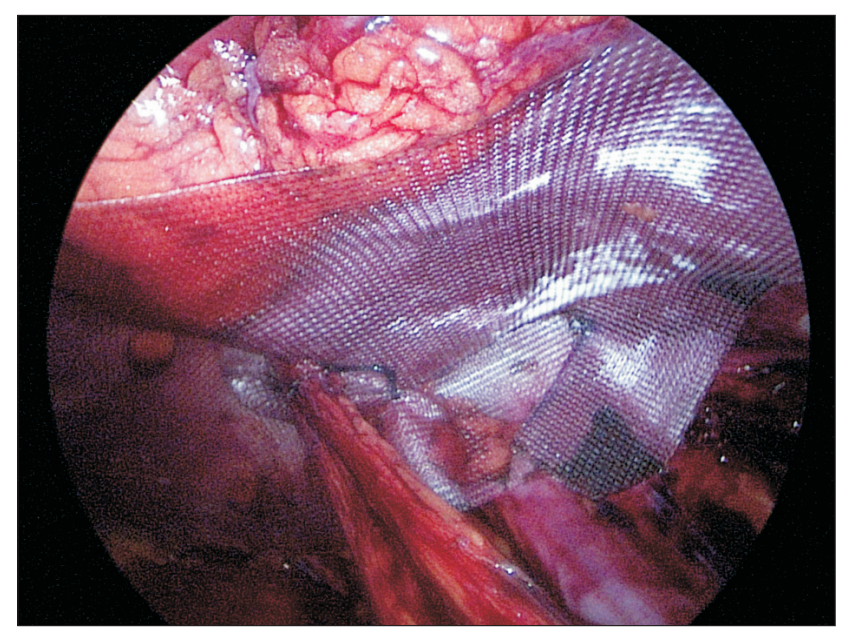

Fig. 4. Encircled and unfolded mesharound cord structure.

ring. After reconstruction of the new internal inguinal ring, we always inserted the tip of the endo-dissector into the new internal inguinal ring to measure the size of the ring, and if needed, we modulated the size of the new internal inguinal ring to prevent a choking effect or a large defect of the encircling mesh around the cord structure (Fig. 2 and 3). After completion of the encircling of the slit, the mesh was unfolded without wrinkles while being cautious about the direction of the mesh (Fig. 4). Also, we were always cautioned against interposing the peritoneum between the mesh and retroperitoneal tissue, and when we slowly desufflated the $\mathrm{CO} 2$ gas from the extraperitoneal space, we carefully inspected the mesh to keep it flat without wrinkling.
Table 1. Characteristics of patients and hernia

\begin{tabular}{cc}
\hline \multicolumn{1}{c}{ Variables } & Values \\
\hline Sex (male : female) & $37: 4(90.2 \%: 9.8 \%)$ \\
Mean age (yr) & 53.5 \\
BMI $\left(\mathrm{kg} / \mathrm{m}^{2}\right)$ & 23.0 \\
Location of hernia & \\
Right & $26(63.4 \%)$ \\
Left & $13(31.7 \%)$ \\
Bilateral & $2(4.9 \%)$ \\
Type of hernia & \\
Unilateral & $39(95.1 \%)$ \\
Direct & 4 \\
Indirect & 33 \\
Both & 3 \\
Bilateral & $2(4.9 \%)$ \\
Indirect + Indirect & 1 \\
Indirect + Direct & 1 \\
Recurrent hernia (direct) & $1(2.4 \%)$ \\
\hline & \\
\hline & \\
\hline
\end{tabular}

Table 2. Perioperative results

\begin{tabular}{|cc}
\hline & Perioperative results \\
\hline Mean operation time & 86.7 min \\
\hline Mean hospital stay & 3.4 days \\
\hline VAS & 3.6 \\
\hline Analgesic use & 1.8 \\
\hline
\end{tabular}

\section{RESULTS}

In this study, there were 37 males and 4 females. The average age was 53.5 years (18 90 years) and the mean body mass index (BMI) was $23.0 \mathrm{~kg} / \mathrm{m}^{2}\left(18.9 \sim 29.3 \mathrm{~kg} / \mathrm{m}^{2}\right)$. The location and type of hernias are listed in detail in Table 1. The mean follow-up period was 23.2 months (12 35 months). Two patients had bilateral inguinal hernia, and one patient had a past history of ipsilateral inguinal hernia surgery using an open method (recurred hernia).

Mean operative time was 86.7 minutes (45 150 minutes). Mean hospital stay was 3.4 days (1 11 days). Postoperative visual analogue scale (VAS) was 3.6 (1 9) and average use of analgesic of hospital day was 1.8 (0 9) (Table 2).

There were no open conversions or other organ injuries during surgery. No cases of established wound infection 
Table 3. Postoperative complications and recurrence

\begin{tabular}{cl}
\hline & Patient \\
\hline Complications & $4(9.8 \%)$ \\
\hline Scrotal discomfort & $3(7.3 \%)$ \\
Scrotal edema & $1(2.4 \%)$ \\
\hline Recurrence & 0 \\
\hline
\end{tabular}

occurred. Three patients complained of scrotal discomfort, and one patient complained about scrotal edema, but they were resolved with conservative management (Table 3). There was no recurrence during the follow up period.

\section{DISCUSSION}

TEP is regarded as the favored method of most types of inguinal hernias ${ }^{9}$ and has the advantages of a shorter hospital stay and an earlier return to work without compromising recurrence. ${ }^{2,3}$ In TEP, different fixation materials have been used, including staples, tacks, or fibrin sealant. Mechanical fixation with staples or tacks has the risk of causing pain or even damage to the surrounding structures such as nerves and vessels. At $20.7 \%$, the prevalence of chronic inguinal pain is higher in the stapled group, whereas that in the fibrin sealant group is $4.7 \%$. $^{10}$ Mesh fixation without a penetrating device (fibrin sealant) did not have a detrimental effect on the quality of life..$^{10,11}$ Also, in our study, there was no chronic groin pain, and there was only intermittent mild chronic discomfort (7.3\%) during the mean 23.2-month follow up. Patients did not need medication or clinical visits. The European Hernia Society recommends that traumatic mesh fixation (non-resorbable devices) in TEP (with a heavyweight mesh) is unnecessary and should be avoided (with exception for some cases like large direct hernias). ${ }^{12}$ However, the fibrin sealant also induces additional costs. Several studies about TEP without a fixing material have been reported and have shown good results. ${ }^{5,13-17}$ If mesh fixation is not used, a surgeon's concern about the complication of mesh migration would not disappear, ${ }^{6-8}$ and if the surgeon is not experienced, anxiety about complications of mesh displacement might be a major problem. Actually, we have experienced two cases of recurrence while using TEP with covering mesh. So we got an idea from Lichtenstein tension free hernia repair and have developed a new method. We performed TEP with the mesh encircling around the cord structure instead of a simple mesh covering over the cord structure, and the cord structure played a role in fixation. In women, we preserved the round ligament to play a fixing role like the spermatic cord. The mesh encircling the cord structure is a factor for the prevention of mesh migration. ${ }^{17}$ Like our study of non-fixation, a result of the study of encircling the cord structure with a preformed mesh showed a good outcome. ${ }^{17}$ But, the method of using a preformed mesh has limitations in that only a designed mesh is used, so a surgeon cannot modify the design, and the preformed mesh costs more than a non-preformed one. The suggested method of mesh design in our study could be modified in response to practical demand according to hernia size, hernia type, etc.

Since the keyhole of the mesh slit could be a route of hernia recurrence, the keyhole should be covered by an additional mesh. ${ }^{17,18}$ We made the mesh slit in the lateroinferior direction and closed the keyhole by a simple overlay suture. There was no recurrence in the mean 23.2-month (12 35 month) follow ups, suggesting that the overlay suture for closure of the keyhole is a good method of preventing hernia recurrence.

If a new internal inguinal ring formed after the overlay suture closure of the slit is very tight on the spermatic cord, it would cause an ischemic injury to the testis. ${ }^{19}$ We always checked during surgery that there was no strangulation of the spermatic cord by mesh wrapping, and there was no ischemic injury of the testis. Inversely, the probability of hernia recurrence would be increased if the new internal inguinal ring is large. We measured the space between the mesh and the cord structure using the endo-dissector tip during mesh wrapping. If the size of the new internal inguinal ring seemed wide or narrow, we controlled the size by repositioning the overlay suture.

This study was limited in that the data was retrospectively reviewed, and the accuracy of hernia recurrence could have been underestimated since we did not directly examine the patients, but called them to determine hernia recurrence, and the period of follow up (23.2 months) was not long term.

In conclusion, the method of mesh-encircling TEP without fixation material and no additional mesh in which the slit of the mesh is wrapped around the cord structure using an overlay suture is a simple and safe technique without compromising recurrence or chronic pain.

\section{REFERENCES}

1) Bracale U, et al. Which is the best laparoscopic approach for inguinal hernia repair: TEP or TAPP? A systematic review of the literature with a network meta-analysis. Surg Endosc 2012;26(12): 3355-3366.

2) Kuhry E, et al. Open or endoscopic total extraperitoneal inguinal hernia repair? A systematic review. Surg Endosc 2007;21(2):161166.

3) Langeveld HR, et al. Total extraperitoneal inguinal hernia repair compared with Lichtenstein (the LEVEL-Trial): a randomized 
controlled trial. Ann Surg 2010;251(5):819-824.

4) Teng YJ, et al. A meta-analysis of randomized controlled trials of fixation versus nonfixation of mesh in laparoscopic total extraperitoneal inguinal hernia repair. Surg Endosc 2011;25(9): 2849-2858.

5) Garg P, et al. Mesh fixation compared to nonfixation in total extraperitoneal inguinal hernia repair: a randomized controlled trial in a rural center in India. Surg Endosc 2011;25(10):3300-3306.

6) Yilmaz I, et al. A rare cause of mechanical bowel obstruction: mesh migration. Hernia 2013;17(2):267-269.

7) Chen MJ, Tian YF. Intraperitoneal migration of a mesh plug with a small intestinal perforation: report of a case. Surg Today 2010;40(6):566-568.

8) Hamouda A, et al. Mesh erosion into the urinary bladder following laparoscopic inguinal hernia repair; is this the tip of the iceberg? Hernia 2010;14(3):317-319.

9) Dulucq JL, Wintringer P, Mahajna A. Laparoscopic totally extraperitoneal inguinal hernia repair: lessons learned from 3,100 hernia repairs over 15 years. Surg Endosc 2009;23(3):482-486.

10) Schwab R, et al. Less chronic pain following mesh fixation using a fibrin sealant in TEP inguinal hernia repair. Hernia 2006;10(3):272-277.

11) Shah NS, Bandara AI, Sheen AJ. Clinical outcome and quality of life in 100 consecutive laparoscopic totally extra-peritoneal (TEP) groin hernia repairs using fibrin glue (TisseelTM): a United
Kingdom experience. Hernia 2012;16(6):647-653.

12) Miserez M, et al. Update with level 1 studies of the European Hernia Society guidelines on the treatment of inguinal hernia in adult patients. Hernia 2014;18(2):151-163.

13) Taylor $C$, et al. Laparoscopic inguinal hernia repair without mesh fixation, early results of a large randomised clinical trial. Surg Endosc 2008;22(3):757-762.

14) Ismail $M$, Garg P. Laparoscopic inguinal total extraperitoneal hernia repair under spinal anesthesia without mesh fixation in 1,220 hernia repairs. Hernia 2009;13(2):115-119.

15) Morrison JE, Jacobs VR. Laparoscopic preperitoneal inguinal hernia repair using preformed polyester mesh without fixation: prospective study with 1-year follow-up results in a rural setting. Surg Laparosc Endosc Percutan Tech 2008;18(1):33-39.

16) Garg $P$, et al. Laparoscopic total extraperitoneal inguinal hernia repair with nonfixation of the mesh for 1,692 hernias. Surg Endosc 2009;23(6):1241-1245.

17) Messaris E, Nicastri G, Dudrick SJ. Total extraperitoneal laparoscopic inguinal hernia repair without mesh fixation: prospective study with 1-year follow-up results. Arch Surg 2010;145(4):334338.

18) Felix E, et al. Causes of recurrence after laparoscopic hernioplasty. A multicenter study. Surg Endosc 1998;12(3):226-231.

19) Chu L, Averch TD, Jackman SV. Testicular infarction as a sequela of inguinal hernia repair. Can J Urol 2009;16(6):4953-4954. 